\title{
Relationships among nasal resistance, age and anthropometric parameters of the nose during growth*
}

\author{
Kaspars Peksis'1,2, Juliane Unger', Santa Paulauska', Aja Emsina' , Martins \\ Blumbergs ${ }^{1}, K^{\prime}$ laus Vogt ${ }^{1}$, Klaus-Dieter Wernecke ${ }^{3}$ \\ ' University of Latvia, Faculty of Medicine, Center of Experimental Surgery, Kalnciema lela 98, LV-1046 Riga, Latvia \\ ${ }^{2}$ Klinika Headline, Kalnciema 98, LV-1046 Riga, Latvia \\ ${ }^{3}$ Sostana Gmbh, Wildensteiner Str. 27, 10318 Berlin, Germany
}

Rhinology Online, Vol 1: 112 - 121, 2018

http://doi.org/10.4193/RHINOL/18.032

*Received for publication:

July 4, 2018

Accepted: September 15, 2018

Published: September 26, 2018

\begin{abstract}
Background: Children generally have a higher nasal resistance than adults. Growth changes the size and different anthropometric parameters of the nose. Logarithmic effective resistance and logarithmic vertex resistance were introduced as physically correct parameters for nasal obstruction. The previously published classification of obstruction derived from 36,500 measurements is missing data for patients aged 7 to 19 years.
\end{abstract}

Methodology: Rhinomanometry was performed before and after decongestion with 9 different anthropometric measurements in 225 children and adolescents. Correlations among age, anthropometric measurements, and logarithmic effective and vertex resistance were determined for both sexes, and regressions were calculated.

Results: The highest correlations with the resistance values were found between age, lateral nasal length, and logarithmic effective resistance. A highly significant linear regression between age and logarithmic effective resistance was also found. This was used for adaption of the classification of obstruction in adults to growing patients. The resistance of the nasal airways at the age of 7 years was about twice that in adults.

Conclusions: The linear regression equations can be used to suborder obstructions measured by four-phase rhinomanometry into classes for estimation of their severity according to age.

Key words: nasal obstruction, 4-phase rhinomanometry, classification, growing age

\section{Introduction}

Since the time of Leonardo da Vinci and Albrecht Dürer, many parameters providing a numerical description of the role of the nose in human facial aesthetic function have been established. Additionally, the changes in these parameters during growth have been extensively described ${ }^{(1-4)}$. A visually 'perfect' nose is not required for optimal function. The morbidity, indications, and techniques of nasal surgery are generally different between children and adolescents, and an unsuccessful surgical outcome has life-long consequences. Therefore, the degree of obstruction of the nasal air stream should be the first consideration in the indications for nasal surgery.

Four-phase rhinomanometry was introduced in 1994 as a me- thodical improvement of 'classic' rhinomanometry according to the 1984 standard of the International Standardization Committee on the Objective Assessment of the Nasal Airway (ISCOANA)

$(5,6)$. The basics and practice of this method are extensively described in Supplement 21 of the journal Rhinology ${ }^{(7)}$. The essentials of four-phase rhinomanometry are the replacement of estimations by correct measurements of pressure and flow, the introduction of new parameters related to the subjective sensing of obstruction, and the acquisition of graphical information about the disturbed function of the nasal valve. Comprehensive clinical material of 36,500 unilateral measurements, a classification of nasal obstruction, and statistically significant data regarding the calculated total nasal resistance were published 


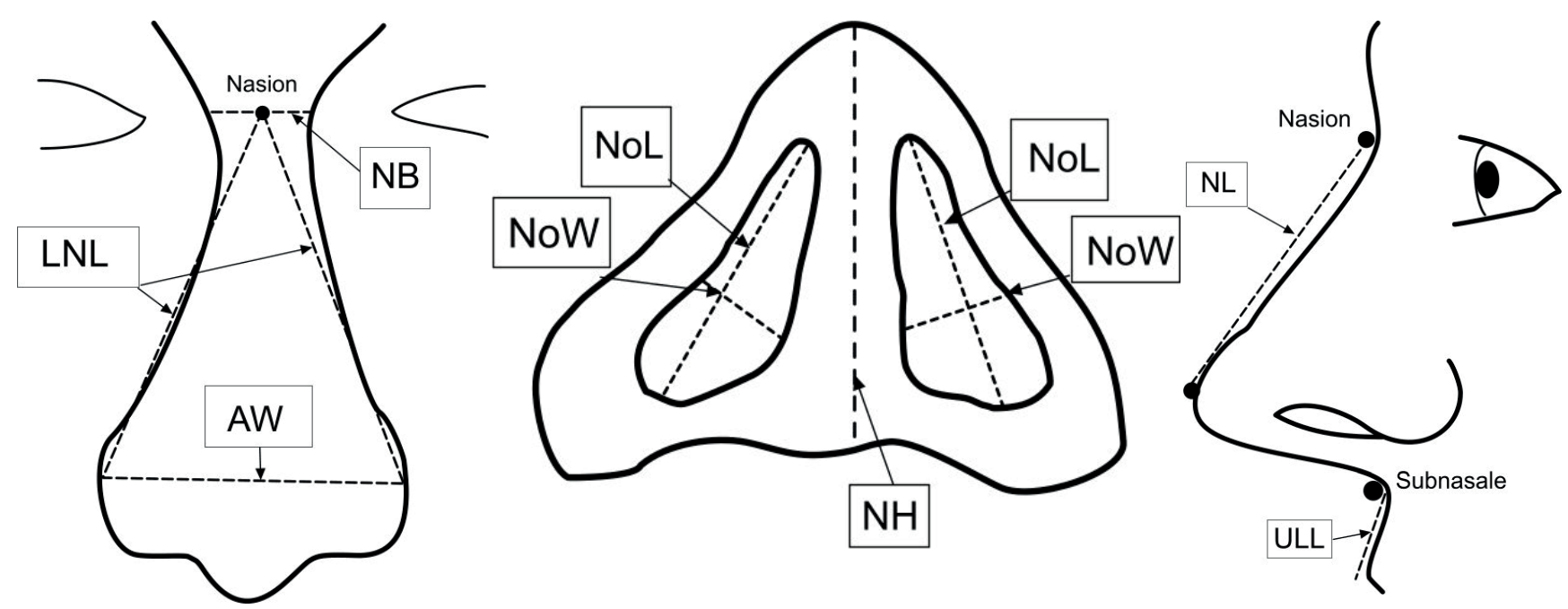

Figure 1. Growth-dependent anthropometric parameters in males and females. NB, nasal base; AW, alar width; LNL, lateral nasal length; ULL, upper lip length; NoW, nostril width; NH, nasal height; NoL, nostril length; NL, nasal length.

in 2015 and $2016^{(8,9)}$. The measurement methods and the correctness of the parameters in four-phase rhinomanometry were confirmed at the 2016 ISCOANA conference and now represent the new standard in rhinomanometry ${ }^{(10)}$. The current classification of nasal obstruction in adults is only valid for the Caucasian population.

Children generally have higher nasal resistance than adults. Growth changes the size and anthropometric parameters of the nose. Additionally, the rate of trivial infections is higher in children, mainly because of the influence of the adenoids. These features of children implicate that the classification of nasal obstruction must be corrected according to the patient's age or the parameters that typically change during growth.

In the present study, we investigated the influence of age and growth on parameters characterising nasal resistance and developed a method with which to adapt rhinomanometric values and the existing classification according to age.

\section{Materials and Methods}

This study was approved by the Ethics Committee for Clinical Research at the University of Latvia (No. 260912-8L). In total, 225 children and adolescents (90 male, 135 female) with no history or symptoms of acute or chronic diseases of the nasal airway were investigated by four-phase rhinomanometry either within a classroom or within a specialised outpatient clinic. All patients' parents provided written informed consent. Patients suspected to have active nasal disease were examined by an ear, nose, and throat surgeon before rhinomanometry. Active anterior rhinomanometry was carried out on both nasal sides using a four-phase rhinomanometer (HRR3 or 4RHINO; Rhinolab, Freiburg, Germany) with software version 4.31 or 5.01 . The coupling of the pressure tube was performed exclusively by tape to preserve the motility of the nasal valve. All patients remained in a sitting position in a classroom at room temperature. The children's position allowed them to see their 'breathing waves' on the computer screen, and all were very cooperative during the procedure. Hence, the data contained no missing results caused by technical errors.

The investigations were repeated $10 \mathrm{~min}$ after application of $0.05 \%$ xylometazoline spray without benzalkonium chloride as a decongestant in the 7- to 10-year-old group (Group A) and with $0.1 \%$ xylometazoline spray in the 11 - to 15 -year-old and 16 - to 19-year-old groups (Groups B and C, respectively). In total, 876 measurements were performed ${ }^{(11-13)}$.

Therefore, within the concept of analysing the nasal air stream in growing children, it was necessary to identify parameters related to the size of the nasal air channel. We selected the parameters listed in Table 1 and depicted in Figure 1; i.e., nasal base, alar width, lateral nasal length, head circumference, upper lip length, nostril width, nasal height, nostril length, and nasal length. These parameters are easy to measure with high intraindividual and inter-individual reproducibility for clinical use or further studies.

For statistical elaboration the measured rhinomanometric data were transferred to Excel tables by the export function of the HRR3 or 4RHINO program. The entire data analysis was carried out using the program SPSS version 22 (IBM Corp., Armonk, NY, USA).

\section{Results}

Step 1: Determination of anthropometric parameters

The descriptive statistics for the anthropometric measurements are summarised in Table 1, and the most important parameters obtained in the three different age groups are shown in Figure 2. The analysis of the age dependency of single parameters clearly 


\begin{tabular}{|c|c|c|c|c|c|c|c|c|c|c|c|c|c|c|c|c|c|}
\hline \multirow[b]{2}{*}{ Age (y) } & & \multicolumn{8}{|c|}{ Males } & \multicolumn{8}{|c|}{ Females } \\
\hline & & NL & NH & NB & ULL & AW & LNL & NoL & Now & NL & NH & NB & ULL & AW & LNL & NoL & Now \\
\hline \multirow{3}{*}{7} & Mean & 3,30 & 1,35 & 1,31 & 1,24 & 2,88 & 3,40 & 1,04 & 0,75 & 2,95 & 1,38 & 1,23 & 1,53 & 2,58 & 3,03 & 1,03 & 0,70 \\
\hline & SD & 0,20 & 0,21 & 0,09 & 0,18 & 0,51 & 0,25 & 0,21 & 0,11 & 0,24 & 0,33 & 0,05 & 0,32 & 0,19 & 0,23 & 0,16 & 0,17 \\
\hline & $\mathrm{n}$ & 11 & 11 & 11 & 11 & 11 & 11 & 11 & 11 & 8 & 8 & 8 & 8 & 8 & 8 & 8 & 8 \\
\hline \multirow{3}{*}{8} & Mean & 3,30 & 1,20 & 1,40 & 1,10 & 2,60 & 3,35 & 1,50 & 0,60 & 3,00 & 1,20 & 1,40 & 1,00 & 2,50 & 3,05 & 1,00 & 0,90 \\
\hline & SD & 0,00 & 0,00 & 0,00 & 0,00 & 0,00 & 0,00 & 0,00 & 0,00 & 0,00 & 0,00 & 0,00 & 0,00 & 0,00 & 0,00 & 0,00 & 0,00 \\
\hline & $n$ & 2 & 2 & 2 & 2 & 2 & 2 & 2 & 2 & 2 & 2 & 2 & 2 & 2 & 2 & 2 & 2 \\
\hline \multirow{3}{*}{9} & Mean & 3,77 & 1,33 & 1,53 & 1,47 & 2,83 & 3,83 & 1,04 & 0,80 & 3,78 & 1,33 & 1,56 & 1,41 & 2,73 & 3,83 & 1,16 & 0,84 \\
\hline & SD & 0,40 & 0,16 & 0,24 & 0,25 & 0,23 & 0,39 & 0,16 & 0,12 & 0,37 & 0,25 & 0,26 & 0,12 & 0,25 & 0,37 & 0,23 & 0,22 \\
\hline & $\mathrm{n}$ & 14 & 14 & 14 & 14 & 14 & 14 & 14 & 14 & 18 & 18 & 18 & 18 & 18 & 18 & 18 & 18 \\
\hline \multirow{3}{*}{10} & Mean & $3 ., 82$ & 1,59 & 1,79 & 1,75 & 2,74 & 3,80 & 1,10 & 0,98 & 3,98 & 1,67 & 1,57 & 1,46 & 2,88 & 4,15 & 1,24 & 0,96 \\
\hline & SD & 0,48 & 0,32 & 0,71 & 0,42 & 0,29 & 0,45 & 0,16 & 0,14 & 0,33 & 0,31 & 0,18 & 0,22 & 0,32 & 0,44 & 0,27 & 0,32 \\
\hline & $\mathrm{n}$ & 7 & 7 & 7 & 7 & 7 & 7 & 7 & 7 & 24 & 24 & 24 & 24 & 24 & 24 & 24 & 24 \\
\hline \multirow{3}{*}{11} & Mean & 4,09 & 1,93 & 1,72 & 1,70 & 3,22 & 3,98 & 1,30 & 0,96 & 4,00 & 1,92 & 1,60 & 1,47 & 3,25 & 4,23 & 1,20 & 0,89 \\
\hline & SD & 0,58 & 0,26 & 0,25 & 0,33 & 0,37 & 0,25 & 0,27 & 0,19 & 0,21 & 0,20 & 0,22 & 0,26 & 0,24 & 0,25 & 0,12 & 0,16 \\
\hline & $n$ & 8 & 8 & 8 & 8 & 8 & 8 & 8 & 8 & 26 & 26 & 26 & 26 & 26 & 26 & 26 & 26 \\
\hline \multirow{3}{*}{12} & Mean & 3,71 & 1,78 & 1,74 & 1,74 & 3,16 & 3,86 & 1,32 & 1,02 & 4,14 & 2,01 & 1,66 & 1,34 & 3,22 & 4,29 & 1,14 & 0,87 \\
\hline & SD & 0,24 & 0,36 & 0,32 & 0,22 & 0,43 & 0,26 & 0,26 & 0,19 & 0,36 & 0,21 & 0,28 & 0,28 & 0,25 & 0,38 & 0,29 & 0,16 \\
\hline & $\mathrm{n}$ & 12 & 12 & 12 & 12 & 12 & 12 & 12 & 12 & 42 & 42 & 42 & 42 & 42 & 42 & 42 & 42 \\
\hline \multirow{3}{*}{13} & Mean & 4,30 & 1,84 & 1,61 & 1,64 & 3,22 & 4,63 & 1,19 & 0,65 & 4,30 & 1,90 & 1,56 & 1,38 & 3,22 & 4,35 & 1,22 & 0,87 \\
\hline & SD & 0,56 & 0,33 & 0,05 & 0,22 & 0,31 & 0,45 & 0,17 & 0,11 & 0,40 & 0,22 & 0,16 & 0,18 & 0,22 & 0,39 & 0,16 & 0,15 \\
\hline & $\mathrm{n}$ & 8 & 8 & 8 & 8 & 8 & 8 & 8 & 8 & 32 & 32 & 32 & 32 & 32 & 32 & 32 & 32 \\
\hline \multirow{3}{*}{14} & Mean & 4,82 & 2,09 & 1,52 & 1,50 & 3,40 & 4,88 & 1,40 & 0,77 & 4,52 & 2,25 & 1,63 & 1,38 & 3,25 & 4,66 & 1,37 & 0,73 \\
\hline & SD & 0,32 & 0,23 & 0,19 & 0,20 & 0,29 & 0,35 & 0,17 & 0,21 & 0,35 & 0,28 & 0,15 & 0,14 & 0,22 & 0,25 & 0,16 & 0,24 \\
\hline & $n$ & 22 & 22 & 22 & 22 & 22 & 22 & 22 & 22 & 18 & 18 & 18 & 18 & 18 & 18 & 18 & 18 \\
\hline \multirow{3}{*}{15} & Mean & 4,90 & 1,93 & 1,47 & 1,53 & 3,54 & 5,06 & 1,44 & 0,71 & 4,75 & 1,90 & 1,48 & 1,32 & 3,20 & 4,62 & 1,26 & 0,61 \\
\hline & SD & 0,53 & 0,26 & 0,04 & 0,31 & 0,32 & 0,22 & 0,18 & 0,20 & 0,49 & 0,24 & 0,18 & 0,17 & 0,16 & 0,18 & 0,14 & 0,09 \\
\hline & $\mathrm{n}$ & 14 & 14 & 14 & 14 & 14 & 14 & 14 & 14 & 18 & 18 & 18 & 18 & 18 & 18 & 18 & 18 \\
\hline \multirow{3}{*}{16} & Mean & 4,52 & 1,83 & 1,92 & 1,51 & 3,24 & 4,75 & 1,28 & 0,65 & 4,35 & 1,85 & 1,85 & 1,30 & 2,95 & 4,36 & 1,18 & 0,55 \\
\hline & SD & 0,33 & 0,33 & 0,15 & 0,16 & 0,41 & 0,32 & 0,17 & 0,15 & 0,32 & 0,35 & 0,13 & 0,20 & 0,19 & 0,40 & 0,13 & 0,10 \\
\hline & $n$ & 22 & 22 & 22 & 22 & 22 & 22 & 22 & 22 & 22 & 22 & 22 & 22 & 22 & 22 & 22 & 22 \\
\hline \multirow{3}{*}{17} & Mean & 4,50 & 1,88 & 1,98 & 1,38 & 3,28 & 4,71 & 1,38 & 0,55 & 4,13 & 1,78 & 1,96 & 1,36 & 3,00 & 4,38 & 1,26 & 0,51 \\
\hline & SD & 0,30 & 0,23 & 0,14 & 0,25 & 0,23 & 0,19 & 0,16 & 0,09 & 0,30 & 0,20 & 0,21 & 0,15 & 0,14 & 0,33 & 0,19 & 0,07 \\
\hline & $\mathrm{n}$ & 22 & 22 & 22 & 22 & 22 & 22 & 22 & 22 & 20 & 20 & 20 & 20 & 20 & 20 & 20 & 20 \\
\hline \multirow{3}{*}{18} & Mean & 4,71 & 1,96 & 1,99 & 1,45 & 3,25 & 4,86 & 1,37 & 0,62 & 4,34 & 1,83 & 1,93 & 1,44 & 3,00 & 4,53 & 1,21 & 0,52 \\
\hline & SD & 0,42 & 0,27 & 0,18 & 0,22 & 0,29 & 0,29 & 0,14 & 0,09 & 0,39 & 0,16 & 0,15 & 0,17 & 0,30 & 0,28 & 0,16 & 0,13 \\
\hline & $n$ & 20 & 20 & 20 & 20 & 20 & 20 & 20 & 20 & 20 & 20 & 20 & 20 & 20 & 20 & 20 & 20 \\
\hline \multirow{3}{*}{19} & Mean & 4,68 & 1,86 & 1,98 & 2,00 & 3,13 & 4,84 & 1,39 & 0,64 & 4,35 & 1,69 & 1,94 & 1,36 & 2,81 & 4,54 & 1,19 & 0,62 \\
\hline & SD & 0,38 & 0,32 & 0,22 & 0,38 & 0,30 & 0,19 & 0,16 & 0,17 & 0,27 & 0,23 & 0,15 & 0,18 & 0,33 & 0,21 & 0,19 & 0,12 \\
\hline & $n$ & 19 & 19 & 19 & 19 & 19 & 19 & 19 & 19 & 20 & 20 & 20 & 20 & 20 & 20 & 20 & 20 \\
\hline
\end{tabular}




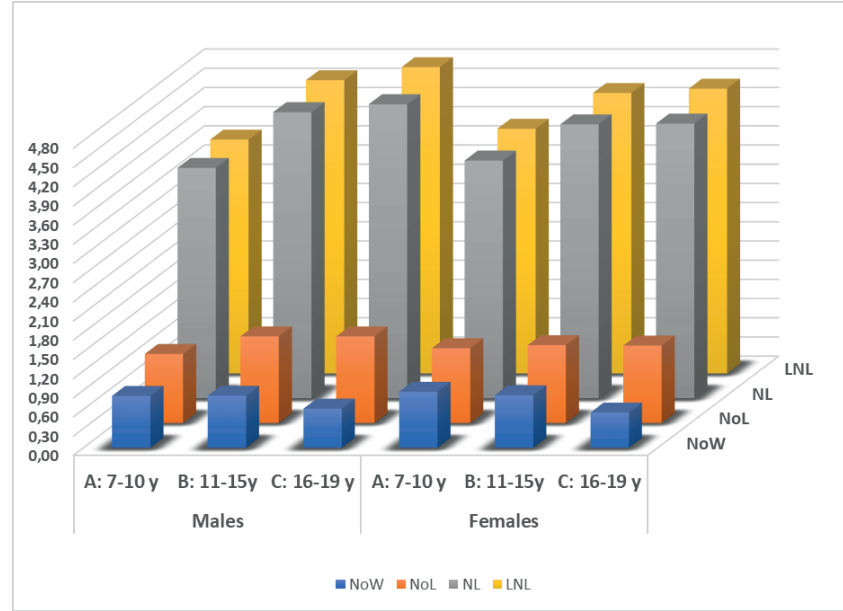

Figure 2. Growth dependence of main anthropometric parameters in males and females. NoW, nostril width; NoL, nostril length; NL, nasal length; LNL, lateral nasal length.

shows that the highest increments were measured for nasal length and lateral nasal length, followed by nasal height. The nasal base and upper lip length showed lower absolute increments because of their minor dimensions. The nostril dimensions seemed to be almost finally determined at the age of 6 to 8 years. These observations are generally well known because the length and height of the nose preeminently determine its size and aesthetic role in the midface. It is also of interest that the length of the nostril increases during the growing period, while the width is smaller in adults as in children. We observed this tendency in both sexes. The differences in the nasal length and lateral nasal length were higher between Groups A and B than between Groups B and C.

To select an anthropometric parameter that was alternatively suitable for adaption of the resistance classification to age, it was necessary to identify the best correlation between age and these parameters.

The best correlation was between the lateral nasal length and

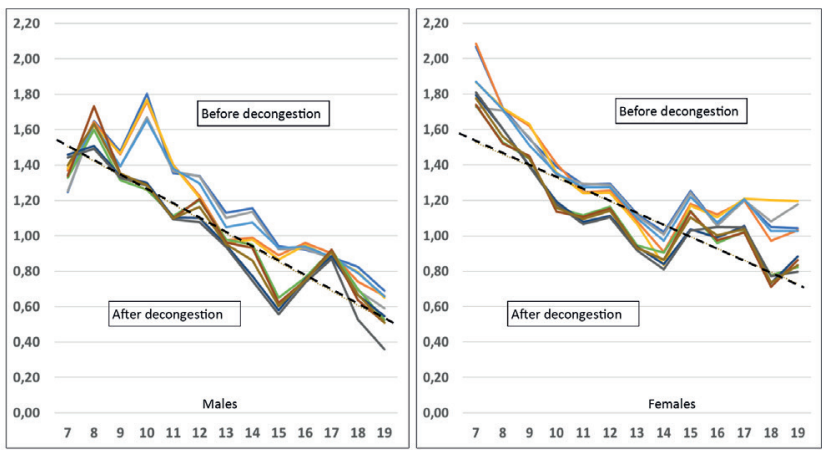

Figure 3. Age-dependent decrease in logarithmic vertex resistance and effective resistances.

age. The lateral nasal length is also the parameter that can be most easily measured.

\section{Step 2: Determination of rhinomanometric parameters} (four-phase rhinomanometry)

The aim of this investigation was to extend the classification of the resistances measured by four-phase rhinomanometry from adults to the age of 7 to 19 years. Previous studies have shown that one-point measurements of the resistance at 75,10 , or 150 $\mathrm{Pa}$ are remnants of the graphical evaluation of rhinomanometric curves and that these values are not related to the subjective sensing of obstruction. Therefore, we followed the new standard (10) in the present study, restricting the parameters to those for which a classification of obstruction in adults was published. The derivation of the parameters has been previously described ${ }^{(3)}$. The following parameters were used: logarithmic vertex resistance in inspiration and expiration, logarithmic effective resistance in inspiration and expiration, and logarithmic effective resistance throughout the entire breath.

The descriptive statistics for the rhinomanometric measurements are shown in Table 3A and B. As expected, the mean values for the logarithmic effective resistance during the entire breath as well as for inspiration and expiration separately were very similar. Additionally, the average values for the vertex resis-

Table 2. Correlations between age and anthropometric parameters.

\begin{tabular}{|c|c|c|c|c|c|c|c|c|}
\hline \multicolumn{9}{|c|}{ Correlations between age and anthropometric parameters } \\
\hline & NL & NH & NB & ULL & AW & LNL & NoL & Now \\
\hline Spearman's rho &, 562 & ,251 &, 580 &, $088^{*}$ & 219 & ,618 & ,317 &, 576 \\
\hline P (two-sided) &, 000 &, 000 &, 000 &, 065 & ,000 & ,000 & ,000 &, 000 \\
\hline Pearson's correlation &, 580 & ,325 &, 558 &, $116^{*}$ & 227 & ,649 & ,302 & ,496 \\
\hline P (two-sided) & ,000 &, 000 &, 000 & ,016 & ,000 &, 000 & ,000 &, 000 \\
\hline
\end{tabular}

Apart from the two p-values with an asterisk, all p value were significant at a level of 0.01 (two-tailed). NL, nasal length; $\mathrm{NH}$, nasal height; $\mathrm{NB}$, nasal base; ULL, upper lip length; AW, alar width; LNL, lateral nasal length; NoL, nostril length; NoW, nostril width. 
Table 3A. Descriptive statistics for resistances at different ages in males.

\begin{tabular}{|c|c|c|c|c|c|c|c|c|c|c|c|}
\hline \multirow{2}{*}{\multicolumn{2}{|c|}{$\begin{array}{l}\text { Males } \\
\text { Age }(y)\end{array}$}} & \multicolumn{5}{|c|}{ Before decongestion } & \multicolumn{5}{|c|}{ After decongestion } \\
\hline & & LVRin & LVRex & LReffin & LReffex & LReff & LVRin & LVRex & LReffin & LReffex & LReff \\
\hline \multirow{3}{*}{7} & Mean & 1,25 & 1,37 & 1,25 & 1,38 & 1,34 & 1,33 & 1,46 & 1,34 & 1,44 & 1,40 \\
\hline & SD & 0,50 & 0,53 & 0,49 & 0,53 & 0,47 & 0,52 & 0,62 & 0,53 & 0,63 & 0,57 \\
\hline & $\mathrm{n}$ & 11 & 11 & 11 & 11 & 11 & 11 & 11 & 11 & 11 & 11 \\
\hline \multirow{3}{*}{8} & Mean & 1,65 & 1,64 & 1,63 & 1,62 & 1,63 & 1,60 & 1,51 & 1,73 & 1,49 & 1,63 \\
\hline & SD & 0,28 & 0,15 & 0,30 & 0,16 & 0,25 & 0,34 & 0,36 & 0,49 & 0,39 & 0,48 \\
\hline & $\mathrm{n}$ & 2 & 2 & 2 & 2 & 2 & 2 & 2 & 2 & 2 & 2 \\
\hline \multirow{3}{*}{9} & Mean & 1,48 & 1,46 & 1,39 & 1,47 & 1,39 & 1,32 & 1,34 & 1,35 & 1,33 & 1,35 \\
\hline & SD & 0,33 & 0,31 & 0,30 & 0,33 & 0,29 & 0,29 & 0,26 & 0,36 & 0,28 & 0,31 \\
\hline & $\mathrm{n}$ & 14 & 14 & 14 & 14 & 14 & 14 & 14 & 14 & 14 & 14 \\
\hline \multirow{3}{*}{10} & Mean & 1,80 & 1,76 & 1,67 & 1,77 & 1,65 & 1,26 & 1,30 & 1,29 & 1,28 & 1,29 \\
\hline & SD & 0,30 & 0,33 & 0,41 & 0,33 & 0,38 & 0,23 & 0,22 & 0,21 & 0,23 & 0,22 \\
\hline & $\mathrm{n}$ & 7 & 7 & 7 & 7 & 7 & 7 & 7 & 7 & 7 & 7 \\
\hline \multirow{3}{*}{11} & Mean & 1,35 & 1,40 & 1,36 & 1,40 & 1,38 & 1,11 & 1,10 & 1,10 & 1,09 & 1,10 \\
\hline & SD & 0,23 & 0,27 & 0,26 & 0,28 & 0,26 & 0,34 & 0,30 & 0,35 & 0,31 & 0,33 \\
\hline & $\mathrm{n}$ & 8 & 8 & 8 & 8 & 8 & 8 & 8 & 8 & 8 & 8 \\
\hline \multirow{3}{*}{12} & Mean & 1,34 & 1,23 & 1,34 & 1,22 & 1,30 & 1,20 & 1,10 & 1,21 & 1,08 & 1,16 \\
\hline & SD & 0,30 & 0,26 & 0,32 & 0,27 & 0,30 & 0,24 & 0,23 & 0,30 & 0,23 & 0,28 \\
\hline & $\mathrm{n}$ & 12 & 12 & 12 & 12 & 12 & 12 & 12 & 12 & 12 & 12 \\
\hline \multirow{3}{*}{13} & Mean & 1,13 & 0,98 & 1,10 & 0,96 & 1,05 & 0,98 & 0,95 & 0,96 & 0,94 & 0,95 \\
\hline & SD & 0,42 & 0,41 & 0,42 & 0,42 & 0,42 & 0,46 & 0,41 & 0,47 & 0,43 & 0,45 \\
\hline & $\mathrm{n}$ & 8 & 8 & 8 & 8 & 8 & 8 & 8 & 8 & 8 & 8 \\
\hline \multirow{3}{*}{14} & Mean & 1,16 & 0,99 & 1,13 & 0,98 & 1,07 & 0,95 & 0,77 & 0,93 & 0,75 & 0,86 \\
\hline & SD & 0,35 & 0,35 & 0,36 & 0,38 & 0,37 & 0,18 & 0,20 & 0,20 & 0,20 & 0,20 \\
\hline & $n$ & 22 & 22 & 22 & 22 & 22 & 22 & 22 & 22 & 22 & 22 \\
\hline \multirow{3}{*}{15} & Mean & 0,94 & 0,89 & 0,93 & 0,87 & 0,93 & 0,65 & 0,58 & 0,62 & 0,56 & 0,60 \\
\hline & SD & 0,42 & 0,42 & 0,43 & 0,43 & 0,38 & 0,23 & 0,23 & 0,24 & 0,23 & 0,23 \\
\hline & $\mathrm{n}$ & 14 & 14 & 14 & 14 & 14 & 14 & 14 & 14 & 14 & 14 \\
\hline \multirow{3}{*}{16} & Mean & 0,92 & 0,96 & 0,93 & 0,95 & 0,94 & 0,76 & 0,76 & 0,75 & 0,74 & 0,75 \\
\hline & SD & 0,44 & 0,42 & 0,47 & 0,44 & 0,45 & 0,27 & 0,28 & 0,30 & 0,28 & 0,28 \\
\hline & $\mathrm{n}$ & 22 & 22 & 22 & 22 & 22 & 22 & 22 & 22 & 22 & 22 \\
\hline \multirow{3}{*}{17} & Mean & 0,88 & 0,90 & 0,87 & 0,87 & 0,88 & 0,92 & 0,89 & 0,92 & 0,87 & 0,91 \\
\hline & SD & 0,40 & 0,39 & 0,41 & 0,40 & 0,40 & 0,34 & 0,32 & 0,36 & 0,33 & 0,34 \\
\hline & $\mathrm{n}$ & 22 & 22 & 22 & 22 & 22 & 22 & 22 & 22 & 22 & 22 \\
\hline \multirow{3}{*}{18} & Mean & 0,82 & 0,74 & 0,69 & 0,80 & 0,79 & 0,70 & 0,67 & 0,64 & 0,53 & 0,67 \\
\hline & SD & 0,31 & 0,34 & 0,50 & 0,54 & 0,33 & 0,27 & 0,18 & 0,18 & 0,27 & 0,23 \\
\hline & $\mathrm{n}$ & 20 & 20 & 20 & 20 & 20 & 20 & 20 & 20 & 20 & 20 \\
\hline \multirow{3}{*}{19} & Mean & 0,69 & 0,66 & 0,59 & 0,65 & 0,66 & 0,53 & 0,55 & 0,51 & 0,36 & 0,51 \\
\hline & SD & 0,36 & 0,34 & 0,61 & 0,73 & 0,35 & 0,22 & 0,20 & 0,21 & 0,16 & 0,21 \\
\hline & $\mathrm{n}$ & 19 & 19 & 19 & 19 & 19 & 19 & 19 & 19 & 19 & 19 \\
\hline
\end{tabular}

SD, standard deviation; LVRin, logarithmic vertex resistance in inspiration; LVRex, logarithmic vertex resistance in expiration; LReffin, logarithmic effective resistance in inspiration; LReffex, logarithmic effective resistance in expiration; LReff, logarithmic effective resistance throughout the entire breath. 
Table 3B. Descriptive statistics for resistances at different ages in females.

\begin{tabular}{|c|c|c|c|c|c|c|c|c|c|c|c|}
\hline \multirow{2}{*}{\multicolumn{2}{|c|}{$\begin{array}{l}\text { Females } \\
\text { Age (y) }\end{array}$}} & \multicolumn{5}{|c|}{ Before decongestion } & \multicolumn{5}{|c|}{ After decongestion } \\
\hline & & LVRin & LVRex & LReffin & LReffex & LReff & LVRin & LVRex & LReffin & LReffex & LReff \\
\hline \multirow{3}{*}{7} & Mean & 2,07 & 2,09 & 1,73 & 1,87 & 1,87 & 1,73 & 1,79 & 1,74 & 1,81 & 1,78 \\
\hline & SD & 0,20 & 0,25 & 0,57 & 0,52 & 0,50 & 0,24 & 0,21 & 0,24 & 0,22 & 0,22 \\
\hline & $\mathrm{n}$ & 8 & 8 & 8 & 8 & 8 & 8 & 8 & 8 & 8 & 8 \\
\hline \multirow{3}{*}{8} & Mean & 1,72 & 1,72 & 1,71 & 1,72 & 1,71 & 1,53 & 1,61 & 1,52 & 1,60 & 1,56 \\
\hline & SD & 0,31 & 0,14 & 0,24 & 0,14 & 0,20 & 0,16 & 0,12 & 0,15 & 0,11 & 0,13 \\
\hline & $\mathrm{n}$ & 2 & 2 & 2 & 2 & 2 & 2 & 2 & 2 & 2 & 2 \\
\hline \multirow{3}{*}{9} & Mean & 1,55 & 1,62 & 1,55 & 1,63 & 1,51 & 1,44 & 1,40 & 1,45 & 1,39 & 1,44 \\
\hline & SD & 0,36 & 0,30 & 0,37 & 0,33 & 0,31 & 0,23 & 0,32 & 0,27 & 0,32 & 0,27 \\
\hline & $\mathrm{n}$ & 17 & 17 & 17 & 17 & 17 & 16 & 16 & 16 & 16 & 16 \\
\hline \multirow{3}{*}{10} & Mean & 1,39 & 1,41 & 1,34 & 1,36 & 1,35 & 1,16 & 1,19 & 1,13 & 1,18 & 1,16 \\
\hline & SD & 0,44 & 0,45 & 0,44 & 0,46 & 0,44 & 0,44 & 0,45 & 0,45 & 0,48 & 0,46 \\
\hline & $\mathrm{n}$ & 24 & 24 & 24 & 24 & 24 & 24 & 24 & 24 & 24 & 24 \\
\hline \multirow{3}{*}{11} & Mean & 1,29 & 1,24 & 1,29 & 1,24 & 1,27 & 1,11 & 1,08 & 1,11 & 1,07 & 1,09 \\
\hline & SD & 0,30 & 0,31 & 0,31 & 0,33 & 0,31 & 0,36 & 0,35 & 0,38 & 0,37 & 0,37 \\
\hline & $\mathrm{n}$ & 26 & 26 & 26 & 26 & 26 & 26 & 26 & 26 & 26 & 26 \\
\hline \multirow{3}{*}{12} & Mean & 1,29 & 1,26 & 1,29 & 1,24 & 1,27 & 1,16 & 1,11 & 1,15 & 1,10 & 1,14 \\
\hline & SD & 0,42 & 0,40 & 0,43 & 0,42 & 0,42 & 0,41 & 0,40 & 0,43 & 0,42 & 0,42 \\
\hline & $\mathrm{n}$ & 42 & 42 & 42 & 42 & 42 & 42 & 42 & 42 & 42 & 42 \\
\hline \multirow{3}{*}{13} & Mean & 1,12 & 1,08 & 1,11 & 1,06 & 1,10 & 0,95 & 0,94 & 0,93 & 0,92 & 0,93 \\
\hline & SD & 0,36 & 0,31 & 0,37 & 0,33 & 0,35 & 0,38 & 0,34 & 0,40 & 0,35 & 0,37 \\
\hline & $\mathrm{n}$ & 32 & 32 & 32 & 32 & 32 & 32 & 32 & 32 & 32 & 32 \\
\hline \multirow{3}{*}{14} & Mean & 1,02 & 0,91 & 1,01 & 0,83 & 0,97 & 0,90 & 0,84 & 0,87 & 0,81 & 0,87 \\
\hline & SD & 0,39 & 0,30 & 0,42 & 0,45 & 0,38 & 0,41 & 0,31 & 0,41 & 0,32 & 0,35 \\
\hline & $\mathrm{n}$ & 18 & 18 & 18 & 18 & 18 & 18 & 18 & 18 & 18 & 18 \\
\hline \multirow{3}{*}{15} & Mean & 1,25 & 1,18 & 1,24 & 1,17 & 1,22 & 1,14 & 1,04 & 1,14 & 1,03 & 1,11 \\
\hline & SD & 0,41 & 0,39 & 0,43 & 0,41 & 0,42 & 0,38 & 0,45 & 0,39 & 0,46 & 0,41 \\
\hline & $\mathrm{n}$ & 18 & 18 & 18 & 18 & 18 & 18 & 18 & 18 & 18 & 18 \\
\hline \multirow{3}{*}{16} & Mean & 1,06 & 1,12 & 1,05 & 1,10 & 1,08 & 0,96 & 0,99 & 0,97 & 1,05 & 1,00 \\
\hline & SD & 0,41 & 0,42 & 0,42 & 0,43 & 0,42 & 0,33 & 0,35 & 0,39 & 0,54 & 0,42 \\
\hline & $\mathrm{n}$ & 22 & 22 & 22 & 22 & 22 & 22 & 22 & 22 & 22 & 22 \\
\hline \multirow{3}{*}{17} & Mean & 1,20 & 1,20 & 1,20 & 1,21 & 1,21 & 1,02 & 1,06 & 1,02 & 1,05 & 1,04 \\
\hline & SD & 0,36 & 0,42 & 0,39 & 0,45 & 0,40 & 0,35 & 0,39 & 0,36 & 0,40 & 0,38 \\
\hline & $\mathrm{n}$ & 20 & 20 & 20 & 20 & 20 & 20 & 20 & 20 & 20 & 20 \\
\hline \multirow{3}{*}{18} & Mean & 1,05 & 0,97 & 1,08 & 1,20 & 1,03 & 0,78 & 0,73 & 0,71 & 0,77 & 0,73 \\
\hline & SD & 0,23 & 0,25 & 0,58 & 0,57 & 0,24 & 0,40 & 0,37 & 0,38 & 0,70 & 0,38 \\
\hline & $\mathrm{n}$ & 13 & 13 & 13 & 13 & 13 & 13 & 13 & 13 & 13 & 13 \\
\hline \multirow{3}{*}{19} & Mean & 1,04 & 1,03 & 1,18 & 1,19 & 1,03 & 0,82 & 0,88 & 0,86 & 0,80 & 0,83 \\
\hline & SD & 0,22 & 0,23 & 0,58 & 0,56 & 0,23 & 0,25 & 0,27 & 0,27 & 0,43 & 0,26 \\
\hline & $\mathrm{n}$ & 13 & 13 & 13 & 13 & 13 & 13 & 13 & 13 & 13 & 13 \\
\hline
\end{tabular}

SD, standard deviation; LVRin, logarithmic vertex resistance in inspiration; LVRex, logarithmic vertex resistance in expiration; LReffin, logarithmic effective resistance in inspiration; LReffex, logarithmic effective resistance in expiration; LReff, logarithmic effective resistance throughout the entire breath. 
Table 4. Pearson's correlations among age, selected anthropometric parameters, and resistances.

\begin{tabular}{|c|c|c|c|c|c|c|c|c|c|c|c|}
\hline & & \multicolumn{10}{|c|}{ Males } \\
\hline & & \multicolumn{5}{|c|}{ Before decongestion } & \multicolumn{5}{|c|}{ After decongestion } \\
\hline & & LVRin & LVRex & LReffin & LReffex & LReff & LVRin & LVRex & LReffin & LReffex & LReff \\
\hline \multirow{3}{*}{ Age } & Correlation coefficient &,- 560 &,- 564 &,- 549 &,- 532 &,- 558 &,- 612 &,- 592 &,- 628 &,- 655 &,- 609 \\
\hline & P (two-sided) & ,000 & ,000 & ,000 &, 000 & ,000 & ,000 & ,000 & ,000 & ,000 & 000 \\
\hline & $\mathrm{n}$ & 181 & 181 & 181 & 181 & 181 & 181 & 181 & 181 & 181 & 181 \\
\hline \multirow{3}{*}{ NL } & Correlation coefficient &,- 379 &,- 446 &,- 355 &,- 433 &,- 400 &,- 458 &,- 511 &,- 448 &,- 522 &,- 487 \\
\hline & P (two-sided) & ,000 & ,000 & ,000 &, 000 & ,000 & ,000 & ,000 & ,000 & ,000 & ,000 \\
\hline & $\mathrm{n}$ & 181 & 181 & 181 & 181 & 181 & 181 & 181 & 181 & 181 & 181 \\
\hline \multirow{3}{*}{ NH } & Correlation coefficient &,$- 157^{*}$ &,- 207 &,$- 117^{*}$ &,- 210 &,$- 155^{*}$ &,- 253 &,- 316 &,- 250 &,- 317 &,- 284 \\
\hline & P (two-sided) & ,034 & ,005 & ,116 & ,005 & ,038 & ,001 & ,000 & ,001 & ,000 & , 000 \\
\hline & $\mathrm{n}$ & 181 & 181 & 181 & 181 & 181 & 181 & 181 & 181 & 181 & 181 \\
\hline \multirow{6}{*}{ LNL } & Correlation coefficient &,- 423 &,- 478 &,- 385 &,- 468 &,- 436 &,- 482 &,- 539 &,- 474 &,- 540 &,- 508 \\
\hline & P (two-sided) & ,000 & ,000 &, 000 & ,000 &, 000 & ,000 & ,000 & ,000 &, 000 & 000 \\
\hline & $\mathrm{n}$ & 181 & 181 & 181 & 181 & 181 & 181 & 181 & 181 & 181 & 181 \\
\hline & & \multicolumn{10}{|c|}{ Females } \\
\hline & & \multicolumn{5}{|c|}{ Before decongestion } & \multicolumn{5}{|c|}{ After decongestion } \\
\hline & & LVRin & LVRex & LReffin & LReffex & LReff & LVRin & LVRex & LReffin & LReffex & LReff \\
\hline \multirow{3}{*}{ Age } & Correlation coefficient &,- 385 &,- 399 &,- 278 &,- 277 &,- 342 &,- 374 &,- 348 &,- 357 &,- 342 &,- 352 \\
\hline & P (two-sided) &, 000 & ,000 & ,000 & ,000 & ,000 & ,000 & ,000 & ,000 & ,000 & ,000 \\
\hline & $\mathrm{n}$ & 255 & 255 & 255 & 255 & 255 & 254 & 254 & 254 & 254 & 254 \\
\hline \multirow{3}{*}{ NL } & Correlation coefficient &,- 457 &,- 476 &,- 407 &,- 446 &,- 436 &,- 426 &,- 451 &,- 418 &,- 442 &,- 432 \\
\hline & P (two-sided) &, 000 & ,000 & ,000 &, 000 & ,000 & ,000 &, 000 & ,000 & ,000 & ,000 \\
\hline & $\mathrm{n}$ & 255 & 255 & 255 & 255 & 255 & 254 & 254 & 254 & 254 & 254 \\
\hline \multirow{3}{*}{ NH } & Correlation coefficient &,- 245 &,- 290 &,- 227 &,- 283 &,- 217 &,- 275 &,- 324 &,- 287 &,- 313 &,- 306 \\
\hline & P (two-sided) & ,000 & ,000 & ,000 & ,000 &, 000 &, 000 &, 000 & ,000 & ,000 &, 000 \\
\hline & $\mathrm{n}$ & 255 & 255 & 255 & 255 & 255 & 254 & 254 & 254 & 254 & 254 \\
\hline \multirow{3}{*}{ LNL } & Correlation coefficient &,- 410 &,- 466 &,- 345 &,- 408 &,- 397 &,- 367 &,- 419 &,- 362 &,- 409 &,- 385 \\
\hline & P (two-sided) &, 000 & ,000 & ,000 & ,000 & ,000 & ,000 & ,000 & ,000 &, 000 & 000 \\
\hline & $\mathrm{n}$ & 255 & 255 & 255 & 255 & 255 & 254 & 254 & 254 & 254 & 254 \\
\hline
\end{tabular}

Apart from the three $p$-values with asterisks, all p-values were significant at a level of 0.01 (two-tailed).

$\mathrm{NL}$, nasal length; NH, nasal height; LNL, lateral nasal length; LVRin, logarithmic vertex resistance in inspiration; LVRex, logarithmic vertex resistance in expiration; LReffin, logarithmic effective resistance in inspiration; LReffex, logarithmic effective resistance in expiration; LReff, logarithmic effective resistance throughout the entire breath.

tance in inspiration and expiration were within the same range; differences in these values appeared after decongestion by $x y-$ lometazoline spray. This was expected and corresponds with our previous investigations in adults ${ }^{(8,9)}$. The values for the effective and vertex resistance only differed when the nasal valve influenced the measurement. The logarithmic effective resistance is the most important parameter for assessment of nasal obstruction, while the vertex resistance is an important parameter when considering the nasal air stream by computational fluid dynamics.
The logarithmic vertex resistance is measured in the steady part of the nasal breathing cycle.

Figure 3 shows the age-dependent variations in resistance in females and males aged 7 to 19 years. The values before and after decongestion are represented by an upper and lower 'bundle' of lines, where every bundle contains the logarithmic mean of vertex resistance in inspiration and expiration as well as the effective resistance in inspiration, expiration, and the entire breath. The dotted line representing trend is very similar between 

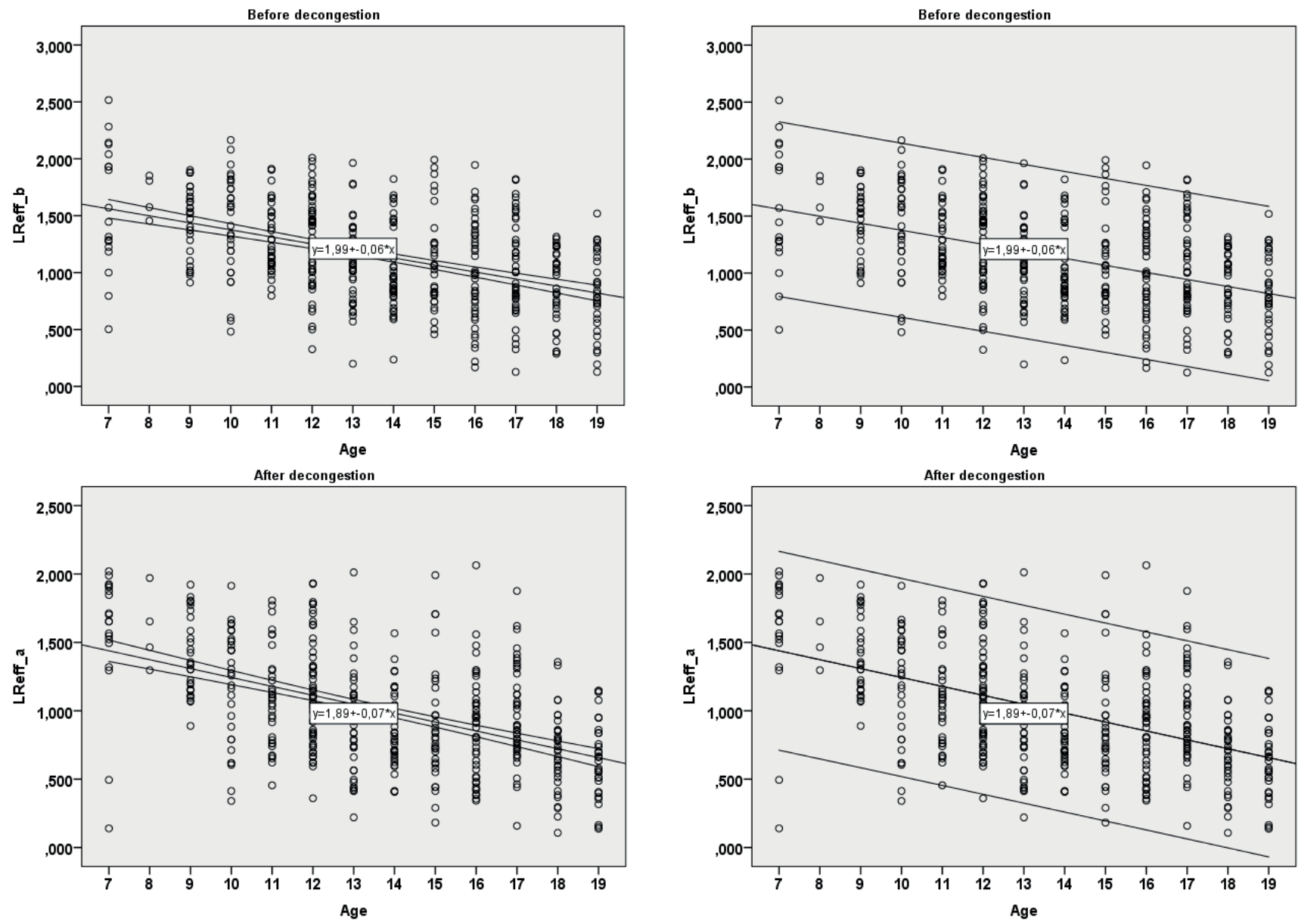

Figure 4. Dependency of logarithmic effective resistance on age before and after decongestion. The left side shows $95 \%$ confidence intervals for mean prediction, and the right side shows $95 \%$ confidence intervals for individual prediction. LReff, logarithmic effective resistance.

the two sexes. The peak at 10 years in non-decongested noses in boys was very likely caused by some measurements performed in patients with undiagnosed infections because it was no longer present after decongestion.

Step 3: Determination of correlations and regressions between age and resistance data

A statistically based enhancement of the resistance classification should be based on the best correlation between age and resistance. A general correlation analysis between age and all resistance parameters was first carried out using Pearson's and Spearman's correlation coefficients.

The correlation with both characteristics was highly significant at a level of $p=0.01$ (two-sided). This result was slightly different between the two sexes. Generally, the values in expiration seemed to correlate better than the values in inspiration. Consequently, we had to conduct a linear regression between one of the resistance parameters and age to obtain a leading correction parameter for enhancement of the classification to children and adolescents.
Figure 4 shows the relationship between logarithmic effective resistance and age before and after decongestion with $95 \%$ confidence intervals for mean as well as individual prediction. The equations for the linear regressions are as follows (both sexes): $y$ [LReff $b]=1.99-0.06^{*} x$ [age] before decongestion and $y$ [LReff_a] $=1.89-0.07^{*} x$ [age] after decongestion, where LReff is the logarithmic effective resistance. Table 5 confirms the linearity of this relationship.

According to the given regressions, the clinical classification of the values for the logarithmic vertex and effective resistance can be adapted and included in the software of rhinomanometers. The resulting correction is reliable for 'normal' noses (bold numbers) because the investigated cohort included only healthy adolescents. However, it may also be useful to estimate the individual degree of obstruction in young patients compared with the values in adults as obtained from the above-mentioned study in adults classifying all degrees of obstructions by 36,500 measurements (last line).

Classifying the 19-year-old group with mean logarithmic effective resistance of 0.93 before decongestion and 0.73 after 
Table 5. Results of the regression analysis (both sexes).

\begin{tabular}{|c|c|c|c|c|c|c|}
\hline \multicolumn{7}{|c|}{ Analysis of Variance } \\
\hline & \multicolumn{3}{|c|}{ LReff before decongestion vs. age } & \multicolumn{3}{|c|}{ LReff after decongestion vs. age } \\
\hline & Sum of squares & $\mathbf{F}$ & $\mathbf{P}$ & Sum of squares & $\mathbf{F}$ & $\mathbf{P}$ \\
\hline Regression & 18.15 & 120.74 & 0.000 & 20.15 & 149.01 & 0.000 \\
\hline Residual & 65.24 & & & 58.56 & & \\
\hline Total & 88.39 & & & 78.71 & & \\
\hline \multicolumn{7}{|c|}{ Coefficients } \\
\hline & \multicolumn{3}{|c|}{ LReff before decongestion vs. age } & \multicolumn{3}{|c|}{ LReff after decongestion vs. age } \\
\hline & B & SE & $\mathbf{P}$ & B & SE & $\mathbf{P}$ \\
\hline Constant & 1.99 & 0.079 & .000 & 1.89 & 0.075 & .000 \\
\hline Age (years) & -0.06 & .006 & .000 & -.070 & .005 & .000 \\
\hline
\end{tabular}

LReff, logarithmic effective resistance; $\mathrm{SE}$, standard error.

decongestion into the classes above places them in the 'green' groups with low or very low resistances. This group closes the gap to the comprehensive clinical classification in Caucasian adults. At the age of 13 years, normal resistances are comparable with class 3 in adults, and at the age of 7 years, the logarithmic resistance in normal children is comparable with class 4 as a clear clinical obstruction in adults. Roughly, the normal resistance in this age is in the range of double that of adults.

The classification of higher obstruction at the age of 7 to 19 years must be confirmed by extended clinical studies because the incidence of morbidities causing nasal obstruction is different from that in adults.

\section{Discussion}

The aim of this study was to identify a relationship between the morphological development of the nose and the functional equivalent of the nasal air stream because previous reports describing this relationship could not be found. Rhinomanometry is the most important measurement method for nasal obstruction, and the rhinologist should be able to compare individual measurements in patients with 'normal values' or better with classes built by comprehensive clinical material. To date, rhinomanometry as a clinical measurement tool during growth has only been used for relative measurement; i.e., it is used to obtain values indicating the timeline of a disease or treatment or to compare resistances before and after decongestion for the differentiation between skeletal and mucosal obstructions.

The anthropometric parameters measured in this study are comparable to those in recent studies of nasal growth (1-3). These studies also showed that the growth velocity is highest between 9 and 14 years. Even at higher ages, growth can change the characteristics of the face. The results of the present study are in agreement with studies of general growth of boys and girls as observed by Buck and Brown ${ }^{(4)}$ and comprehensive growth analyses published by the World Health Organization. The above-mentioned references as well as the analysis of the anthropometric parameters in the present study confirm that the relationship between anthropometric parameters and age is exponential but non-linear.

Quantitative investigations of the nasal respiratory pattern during growth and development were carried out by Laine-Alava and Minkkinen ${ }^{(14)}$, who concluded that guidelines for adults are applicable from 16 years of age on. The present results show that growth-dependent changes can be expected up to the age of 19 years in boys.

We expected to find that the nasal resistance diminishes with increasing dimensions of the nasal airway, but it seems remarkable that the decrease in the logarithmic nasal vertex resistance or logarithmic effective resistance is linear but not exponential. This statistical relationship can be explained by the logarithmic transformation of the resistance values as well as by the fact that the relationship between the nasal cross-sectional area and resistance is determined by the fourth power of the radius (Hagen-Poiseuille law). The linear regression between age and logarithmic resistance allows for easy estimation of the individual severity of obstruction in relation to the obstruction classes in adults.

The classification of obstruction as shown in Table 6 is more reliable for the 'green' classes (i.e., the healthy noses after decongestion), while classes 3 to 5 are built from material that is much smaller because of the comparative classification in adults from 36,500 measurements ${ }^{(8)}$.

The observation that the lateral nasal length has a very significant correlation with age is important if analyses of the face are carried out before intended corrections of the face by orthodon- 
tic measures or corrective facial surgery.

\section{Acknowledgements}

The authors thank Angela Morben, DVM, ELS, from Edanz Group (www.edanzediting.com/ac), for editing a draft of this manuscript.

Table 6. Enhanced classification of logarithmic resistance values in adolescents.

\begin{tabular}{|c|c|c|c|c|c|}
\hline \multicolumn{6}{|c|}{ Clinical obstruction classes before without decongestion } \\
\hline Age & 1 & 2 & 3 & 4 & 5 \\
\cline { 2 - 7 } $\mathbf{7}$ & $<1.38$ & $\mathbf{1 . 3 8 - 1 . 8 4}$ & $1.84-2.30$ & $2.30-2.76$ & $>2.76$ \\
\hline $\mathbf{8}$ & $<\mathbf{1 . 3 3}$ & $\mathbf{1 . 3 3}-\mathbf{1 . 7 7}$ & $1.77-2.21$ & $2.21-2.66$ & $>2.66$ \\
\hline $\mathbf{9}$ & $<\mathbf{1 . 2 8}$ & $\mathbf{1 . 2 8}-\mathbf{1 . 7 0}$ & $1.70-2.13$ & $2.13-2.55$ & $>2.55$ \\
\hline $\mathbf{1 0}$ & $<\mathbf{1 . 2 2}$ & $\mathbf{1 . 2 2}-\mathbf{1 . 6 3}$ & $1.63-2.03$ & $2.03-2.45$ & $>2.45$ \\
\hline $\mathbf{1 1}$ & $<\mathbf{1 . 1 7}$ & $\mathbf{1 . 1 7}-\mathbf{1 . 5 6}$ & $1.56-1.95$ & $1.95-2.34$ & $>2.34$ \\
\hline $\mathbf{1 2}$ & $<\mathbf{1 . 1 2}$ & $\mathbf{1 . 1 2}-\mathbf{1 . 4 9}$ & $1.49-1.86$ & $1.86-2.24$ & $>2.24$ \\
\hline $\mathbf{1 3}$ & $<\mathbf{1 . 0 7}$ & $\mathbf{1 . 0 7 - 1 . 4 2}$ & $1.42-1.78$ & $1.78-2.13$ & $>2.13$ \\
\hline $\mathbf{1 4}$ & $<\mathbf{1 . 0 1}$ & $\mathbf{1 . 0 1 - 1 . 3 5}$ & $1.35-1.69$ & $1.69-2.025$ & $>2.025$ \\
\hline $\mathbf{1 5}$ & $<\mathbf{0 . 9 6}$ & $\mathbf{0 . 9 6 - 1 . 2 8}$ & $1.28-1.60$ & $1.60-1.92$ & $>1.92$ \\
\hline $\mathbf{1 6}$ & $<\mathbf{0 . 9 1}$ & $\mathbf{0 . 9 1 - 1 . 2 1}$ & $1.21-1.51$ & $1.51-1.81$ & $>1.81$ \\
\hline $\mathbf{1 7}$ & $<\mathbf{0 . 8 6}$ & $\mathbf{0 . 8 6 - 1 . 1 4}$ & $1.14-1.43$ & $1.43-1.71$ & $>1.71$ \\
\hline $\mathbf{1 8}$ & $<\mathbf{0 . 8 0}$ & $\mathbf{0 . 8 0 - 1 . 0 7}$ & $1.07-1.34$ & $1.34-1.61$ & $>1.61$ \\
\hline$>\mathbf{1 8}$ & $\leq \mathbf{0 . 7 5}$ & $\mathbf{0 . 7 5 - 1 . 0 0}$ & $\mathbf{1 . 0 0 - 1 . 2 5}$ & $\mathbf{1 . 2 5}-\mathbf{1 . 5 0}$ & $>\mathbf{1 . 5 0}$ \\
\hline
\end{tabular}

\section{Funding}

This study was funded in part by the ZIM "Rhinodiagnost" project ZF 4428701CS7.

\section{Authorship contribution}

$\mathrm{KP}$ clinical supervisor, anthropometric analysis, writing; JU, $A E$, $\mathrm{SP}$ and $\mathrm{MB}$ investigations and data collection, $\mathrm{KV}$ and K.-D.W. statistical analysis.

\section{Availability of data and materials}

The datasets used and/or analysed during the current study are available from the corresponding author on reasonable request.

\section{Conflict of interest}

The authors declare that they have no competing interests.
1. Zankl A, Eberle L, Molinari L, Schinzel A. Growth charts for nose length, nasal protrusion and philtrum length from birth to 97 years. Am J Med Genet 2002; 111: 388-391.

2. Van der Heyden P, Korsten-Meijer AG, van der Laan BF, Wit HP, Goorhuis-Brouwer SM Nasal growth and maturation age in adolescents: a systematic review. Arch Otolaryngol Head Neck Surg 2008; 134: 1288-1293.

3. Sforza C, Grandi G, De Menezes M, Tartaglia GM. Age- and sex-related changes in the normal human external nose. Forensic Sci Int 2011; 204: 205.e1-9.

4. Buck DL, Brown CM. A longitudinal study of nose growth from ages 6 to 18. Ann Plast Surg 1987; 18: 310-313.

5. Clement P. Committee report on standardization of rhinomanometry. Rhinology 1984; 22: 151-155.

6. Clement P, Gordts F. Consensus report on acoustic rhinometry and rhinomanometry. Rhinology 2005; 43: 169-179.

7. Vogt K, Jalowayski AA, Althaus W, et al. 4-Phase-rhinomanometry (4PR) - basics and practice 2010. Rhinology 2010; Suppl 21: 1-50.
8. Vogt K, Wernecke KD, Behrbohm H, Gubisch W, Argale M. Four-phase rhinomanometry: a multicentric retrospective analysis of 36,563 clinical measurements. Eur Arch Otorhinolaryngol 2016; 273: 1185-1198.

9. Vogt K, Wernecke K-D, Argale M, Kaulina K. Classification of total nasal obstruction in 10,300 cases by 4-phase-rhinomanometry. Rom J Rhinol 2016: 6: 149-160.

10. Vogt K, Bachmann-Harildstad G, Lintermann A, Nechyporenko A, Peters F, Wernecke KD. The new agreement of the international RIGA consensus conference on nasal airway function tests. Rhinology 2018; 56; 2 : 133-143.

11. Unger J. Investigations to normal values in children in 4-phase-rhionomanometry. Diploma thesis. Faculty of Medicine. Riga: University of Latvia, 2011.

12. Emsina A. 4-phase-rhinomanometry. Normal values in adolescents and correlation with anthropometric parameters. Diploma Thesis. University of Latvia, Faculty of Medicine 2013 (Latvian).

13. Blumbergs M. Evaluation of nasal resistance by 4 -phase-rhinomanometry in the age of 16-19 years and the relation between acquired data and anthropometric measurements. Diploma Thesis. University of Latvia, Faculty of Medicine 2018 (Latvian).

14. Laine-Alava MT, Minkkinen UK. Variation of nasal respiratory pattern with age during growth and development. Laryngoscope 1997; 107: 386-390.

15. Shaw RB Jr, Kahn DM. Aging of the midface bony elements: a three-dimensional computed tomographic study. Plast Reconstr Surg 2007; 119: 675-681.

\section{Dr. Kaspars Peksis}

University of Latvia

Faculty of Medicine

Center of Experimental Surgery

Kalnciema lela 98

LV-1046 Riga

Latvia

E-mail: kaspars.peksis@inbox.Iv 